\title{
Development of method for determination of the kinetics constants of coal char gasification in pore diffusion regimes
}

\author{
Irina Remkunas ${ }^{1}$, Igor Donskoy ${ }^{2, *}$, and Aleksandr Kozlov ${ }^{2}$ \\ ${ }^{1}$ Irkutsk State University, Karla Marksa st. 1, Irkutsk, Russia \\ ${ }^{2}$ Melentiev Energy Systems Institute SB RAS, 130 Lermontova st., Irkutsk, Russia
}

\begin{abstract}
In this paper, we developed a method for determining the kinetic constants of partially diffusioncontrolled heterogeneous reactions in a porous sample of powder. Studies have been conducted on the experimental data of thermogravimetric analysis of carbon conversion in a stream of $\mathrm{CO}_{2}$, using a new method of processing kinetic curves, to obtain updated values of the kinetic constants under conditions where widely used models are inappropriate. Data obtained can be used for a reliable assessment of the characteristics of the gasification process.
\end{abstract}

\section{Introduction}

Gasification methods allows the processing of various solid fuels, ranging from peat brown coal and ending with coal and anthracite, in a wide range of chemical composition and other properties. Gasification of solid fuel results in syngas, from which it is possible to obtain other gases for various energy and chemical purposes [1]. Gasification plants could feed chemical plants (for example, ammonia or methanol production) and energy plants (integrated gasification combined cycle plants, IGCC). There are several methods of solid fuel gasification: fixed bed conversion, fluidized bed conversion, entrained flow conversion. Under different conditions (particle size, reactor hydrodynamics, etc.), different regimes of gasification are observed.

There is a large amount of studies on coal gasification using various gasifying agents, such as steam and carbon dioxide. In all studies there is a wide variation of parametric conditions, as well as the use of various equipment.

Recently, authors of [2] studied the thermodynamic and kinetic characteristics of coal gasification using thermodynamic calculations. In the course of these studies, it was revealed that at the early stage the interface reaction is the controlling factor in coal gasification. With an increase in the kinetic coefficient values, the controlling factor gradually changes from interfacial reaction to internal diffusion. At 1173-1473K, the limiting stage of coal gasification is, first of all, the interfacial reaction.

In [3], M. Irfan et al. presented an overview of coal gasification in the presence of $\mathrm{CO}_{2}$ as a reagent. They considered various factors of coal gasification with $\mathrm{CO}_{2}$, including: pressure, temperature, gas composition, catalysts and minerals, heating rate, particle size, and various types of reactors. Different equations for the reaction rate (Arrhenius and Langmuir-Hinshelwood) for gasification of semi-coke in the kinetic control of the reaction (low temperature) and the diffusion control (high temperature) at low and high pressures are considered.

The rate of gasification increases with increasing temperature during catalytic and non-catalytic gasification of the reaction. At low and medium temperatures, a part of mineral substances volatilize or turn into new mineral phases, but in the hightemperature region these minerals do not completely disappear, which in turn leads to a decrease in the activity of the coal sample. In addition, the difference between the gasification rates becomes very small with increasing temperature and is practically not observed at very high temperatures. Thus, after some temperature further heating has no practical meaning. Therefore, researchers develop various catalysts or add a certain amount of mineral substances to coal samples in order to influence the reaction rate at lower temperature ranges.

In the review [4], experimental and theoretical studies of the carbon gasification in various gaseous media and at high temperatures (up to $1373 \mathrm{~K}$ ) are considered. Descriptions of experimental methods, as well as their theoretical descriptions, are presented.

Surface reactions proceed through interphase intermediate compounds, so net kinetics is concerned with active surface reaction centers. The results of their research [4] show that comprehensive description of heterogeneous reaction $\mathrm{C}+\mathrm{CO}_{2}$ is possible on the basis of studying the behavior of surface oxides, which can react in different ways; the true reaction rate does not depend on the partial pressure of $\mathrm{CO}_{2}$ and the degree of conversion. Also, the authors confirm that the net reaction rate does not depend on the type of carbon in the absence of catalytically active additives. 
When studying the conversion of solid fuels, many authors estimate the kinetic characteristics using incorrectly averaged data that leads to unreliable estimate of the kinetic characteristics of the heterogeneous reactions. It is necessary to improve the methods that will allow to obtain reliable and sufficiently accurate data during conversion of solid fuels. Transfer processes and equipment features can influence the results of kinetic measurements $[5,6]$. In this paper, we are trying to reduce transfer effects when studying the $\mathrm{C}$ $+\mathrm{CO}_{2}$ reaction.

\section{Kinetic models of carbon gasification}

Experiments were carried out with carbon conversion in carbon dioxide atmosphere in thermogravimetric analyser NETZSCH STA $449 \mathrm{C}$, the sample was a brown coal char; input gas flows: $10 \mathrm{ml} / \mathrm{min}$ of $\mathrm{Ar}$ and $30 \mathrm{ml} / \mathrm{min}$ of $\mathrm{CO}_{2}$. Experimental techniques are described in $[7,8]$.

Since the chemical reaction occurs at interphase surface, different regimes of its interaction with diffusion are possible [9]. Following models were used during curves processing:

1) at a low chemical reaction rate, the concentration of the reacting substances in the vicinity of the reactive surface is close to the measured concentrations in main flow, so apparent reaction kinetics fully coincides with the true one ("kinetic region") .

2) when the reaction rate is significantly higher than the transport rate, the observed macroscopic reaction kinetics is determined by diffusion and does not reflect the true reaction rate on the surface, its dependence on temperature, concentration of reactants, surface activity, etc. ("diffusion region");

3) Zeldovich [10] and Thiele [11] considered theoretically the third case, when the diffusion rate and chemical reaction rate, considered independently of each other, are comparable. Then there is a transition region in which the following relations hold:

$$
\begin{gathered}
\frac{d m}{d \tau}=-S_{0} C_{C O 2} M_{C} \sqrt{K D S_{P}} \\
\left(\frac{d m}{d \tau}\right)^{2}=\left[S_{0} C_{C O 2} M_{C}\right]^{2} K D S_{P} \\
K=\frac{\left(\frac{d m}{d \tau}\right)^{2}}{D S_{P}\left[S_{0} C_{C O 2} M_{C}\right]^{2}} \\
D=2 * 10^{-5}\left(\frac{T}{273}\right)^{1.5} \\
C_{C O 2}=\frac{P_{0}}{R T} \omega_{C O 2} \\
S_{0}=\frac{\pi}{4} d_{0}^{2} \\
S_{P}=\frac{6(1-\Pi)}{d_{P}} \\
E k_{0}-\frac{E}{R T}=\ln \left\{\frac{\left(\frac{d m}{d \tau}\right)^{2}}{D S_{P}\left[S_{0} C_{C O 2} M_{C}\right]^{2}}\right\}
\end{gathered}
$$

Here $S_{0}$ is crucible section area, $M_{C}-$ carbon molar mass, $C_{\mathrm{CO} 2}$ - carbon dioxide concentration, $K$ - kinetic constant, $D$ - diffusivity, $S_{p}-$ specific pore surface, $\omega_{\mathrm{CO} 2}$ - malar fraction of $\mathrm{CO}_{2}, \Pi$ - porosity, $d_{P}-$ mean pore diameter.

Reaction regimes with internal diffusion-kinetics interaction have Arrhenius dependence on temperature, but apparent activation energy is a half of true activation energy.

\section{Analysis of experimental TG-curves}

According to experimental data on the conversion of carbon in $\mathrm{CO}_{2}$ at heating rate $10 \mathrm{~K} / \mathrm{min}$, curves were processed under different assumptions:

1. The reaction is in the purely kinetic region.

Graphic dependency was built based on following relation:

$$
\ln \left[-\frac{d m}{d \tau} * \frac{1}{f(m)}\right]=f\left(\frac{1}{T}\right)
$$

Different variants of $f(m)$ were substituted to find linear dependence (fig. 1).
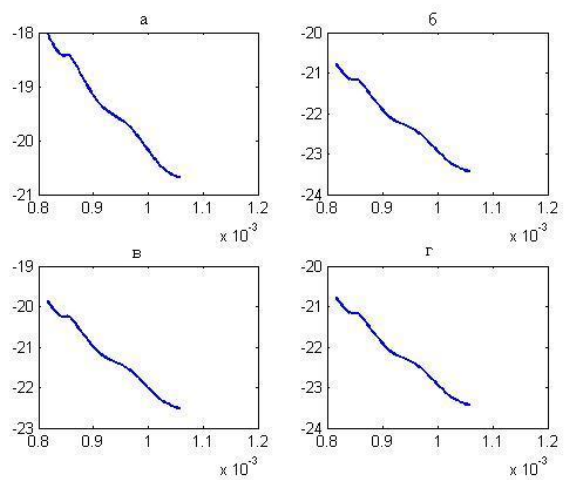

Fig. 1. Arrhenius plots: (a) $f(m)=m^{0}$, (b) $f(m)=m$, (c) $f(m)=m(1-$ $m),\left(\right.$ d) $f(m)=m^{2 / 3}$.

All used approximations of $f(m)$ were close. Given $f(m)=m^{0}$, calculated values of kinetic coefficients are as follows: $E_{a}=96.5 \mathrm{~kJ} / \mathrm{mol}$ and $k_{0}=1.73 \times 10^{-4} \mathrm{~kg} / \mathrm{s}$. Apparent activation energy is very low compared to available published data.

2. If heterogeneous reaction is under diffusion control, then apparent reaction rate depends on temperature in following way:

$$
\ln \left(-\frac{d m}{d \tau}\right)=P \ln T+C
$$

Results of TG-curve processing is presented at fig. 2. Linear approximation coefficient in this relation corresponds to exponent that is in the range of 1-2 for diffusion process [12]. However, calculated value of $P$ is 10.5 , so diffusion model is not applicable in this case.

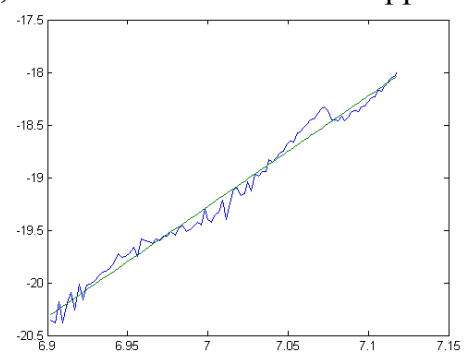

Fig. 2. Dependence $\ln (-d m / d t)=f(\ln T)$

3. Based on foregoing, intermediate regimes should be considered, where rates of kinetics and diffusion are 
compatible. Graphical dependence is plotted at fig. 3a following relation:

$$
\ln \left\{\frac{\left(\frac{d m}{d \tau}\right)^{2}}{D S_{P}\left[S_{0} C_{C O 2} M_{C}\right]^{2}}\right\}=f\left(\frac{1}{T}\right)
$$

Kinetics coefficients are obtained using linear approximation: $E_{a}=201.16 \mathrm{~kJ} / \mathrm{mol}, k_{0}=1.3 * 10^{4} \mathrm{~m} / \mathrm{s}$. These values were used to reproduce experimental TGcurve at fig. 3b: good match is observed between calculated and measured data.
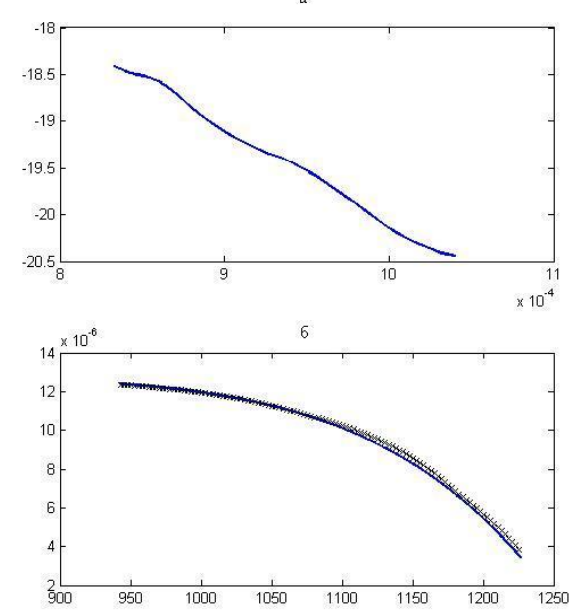

Fig. 3. Heating rate $10 \mathrm{~K} / \mathrm{min}$ : (a) Arrhenius plot for pore diffusion model; (b) - comparison of experimental and calculated TG-curves.

To confirm this method, the curves obtained by analyzing the experimental data at two other heating rates are given. At the heating rate $1 \mathrm{~K} / \mathrm{min}$ (Fig. 4), there is also a linear dependence at Arrhenius plot, and the calculated data match well the experimental curve. However, value of $E_{a}$ drops, its values are again two times lower than expected one, namely $111.9 \mathrm{~kJ} / \mathrm{mol}$. This suggests that reaction moves back to the kinetic region. By calculating $E_{a}$ for this heating rate using the integral method, the value of $200.3 \mathrm{~kJ} / \mathrm{mol}$ was obtained.

For the heating rate $20 \mathrm{~K} / \mathrm{min}$ (Fig. 5), agreement between the calculated curve and the experimental one is not as good as at $10 \mathrm{~K} / \mathrm{min}$ due to features of temperature compensation in the device. The value of $E_{a}$ in this case is $186 \mathrm{~kJ} / \mathrm{mol}$.

\section{Conclusions}

One of the options for processing thermogravimetric curves for kinetic coefficients is considered. The selected method allows to take into account pore diffusion effects. This technique is applicable for medium heating rates when pure kinetic regimes may not be observed.

Using this method kinetic coefficients of carbon gasification were obtained. Activation energy estimated by the developed method, vary from 186 to $201 \mathrm{~kJ} / \mathrm{mol}$, which is consistent with the literature data.
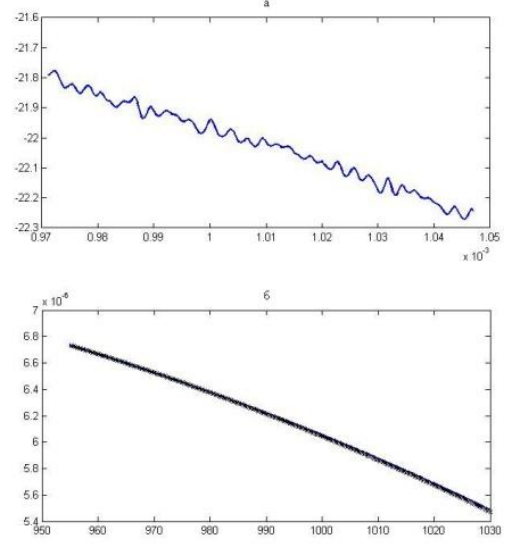

Fig. 4. Heating rate $1 \mathrm{~K} / \mathrm{min}$ : (a) Arrhenius plot for pore diffusion model; (b) - comparison of experimental and calculated TG-curves.
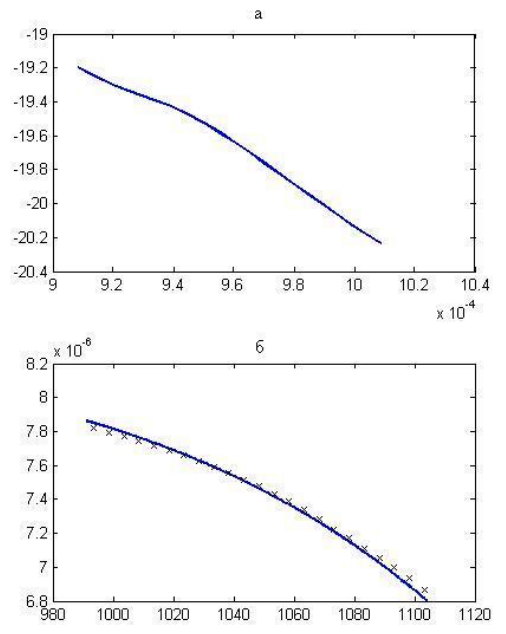

Fig. 5. Heating rate $20 \mathrm{~K} / \mathrm{min}$ : (a) Arrhenius plot for pore diffusion model; (b) - comparison of experimental and calculated TG-curves.

\section{References}

1. C. Higman, S. Tam, Chemical Reviews 114, 1673 (2014). DOI: $10.1021 / \mathrm{cr} 400202 \mathrm{~m}$

2. C. Lan, Q. Lyu, Y. Qie, M. Jiang, X. Liu, S. Zhang, Thermochimica Acta, 666, 174 (2018). DOI: 10.1016/j.tca.2018.06.019

3. M.F. Irfan, M.R. Usman, K. Kusakabe, Energy, 36, 12 (2011).

4. E.S. Golovina, Combustion, Explosion and Shock Waves, 38, 401 (2002).

5. A. Gomez, N. Mahinpey, Fuel, 148, 160 (2015).

6. G.I. Khudyakova, Experimental study on thermochemical conversion of coal chars by thermogravimetric analysis. Cand. Sci. thesis. (Ural Federal Univ., 2015).

7. A. Kozlov, D. Svishchev, I. Donskoy, V. Shamansky, A. Ryzhkov, Journal of Thermal Analysis and Calorimetry, 122, 1213 (2015). 
8. A.N. Kozlov, D.A. Svishchev, G.I. Khudiakova, A.F. Ryzhkov, Solid Fuel Chemistry, 51, 205, (2017).

9. K. Annamalai, W. Ryan, Progress in Energy and Combustion Science, 19, 383, (1993).

10. Ya.B. Zeldovich, Selected works. Vol. 1: Chemical Physics and Hydrodynamics (Princeton Univ. Press, 1992).

11. E.W. Thiele, Ind. Eng. Chem., 31, 916, (1939). https://doi.org/10.1021/ie50355a027

12. T.K. Sherwood, R.L. Pigford, C.R. Wilke, Mass Transfer (McGraw-Hill, 1975). 